\title{
Flow in conical shock waves: A signal for the deconfinement transition?
}

\author{
D. H. Rischke, H. Stöcker, and W. Greiner \\ Institut für Theoretische Physik der J. W. Goethe Universität, Postfach 111932, \\ D-6000 Frankfurt am Main 11, West Germany \\ (Received 18 January 1990)
}

\begin{abstract}
We investigate the hydrodynamical flow of nuclear matter in a conical-shock-wave scenario of a central, asymmetric heavy-ion collision. This work is motivated by a suggestion of Chapline and Granik that the creation of a deconfined phase of quarks and gluons behind the shock will appreciably increase the deflection angle of the matter flow. We employ several hadron matter equations of state recently suggested to solve the conical-shock-wave problem and compare the results with a calculation using the bag equation of state. We find that large differences in the deflection angle obtained in the rest frame of the shock vanish in the laboratory system. However, a signature for the deconfinement transition may be the transverse momentum of the matter flow, which is up to a factor of 2 larger for the quark-gluon plasma. Thus, an excitation function of the mean transverse momentum would show an increase at a certain bombarding energy, signaling the onset of the deconfinement transition.
\end{abstract}

\section{INTRODUCTION}

One of the most intriguing questions of nuclear physics nowadays is how nuclear matter behaves at high densities and temperatures. ${ }^{1-3}$ Heavy-ion collision experiments at various bombarding energies are up to now the only means to probe this behavior far from the nuclear-matter ground state. To extract information for theoretical concepts of strongly interacting matter, one has to compare the experimental results with dynamical models of heavy-ion collisions, which require these concepts as input. Such models may describe the collision in microscopical or macroscopical terms, such as, e.g., the hydrodynamical approach. ${ }^{1-3}$ The appeal of the latter is that properties of nuclear matter are parametrized in terms of macroscopic variables which are easy to interpret and are related by an equation of state (EOS). A great deal of effort has been spent to extract this EOS. ${ }^{1,3}$ Up to now, because of the complexity of quantum chromodynamics (QCD), this EOS is of phenomenological origin.

To justify the application of ideal hydrodynamics to heavy-ion collisions, one assumes that interactions between particles happen on a scale which is small as compared to the system's size. They should also happen sufficiently often and fast to establish local thermodynamical equilibrium. Still, the full $(3+1)$-dimensional problem requires enormous numerical effort. ${ }^{4}$ Therefore, models have been developed which try to appropriately parametrize the actual flow pattern in a collision in simple terms and thus simplify the hydrodynamical equations.

One of them is the one-dimensional shock model, convenient to describe the central region in symmetric headon collisions. ${ }^{5}$ For a central collision of a small projectile with a large target, the conical-shock-wave model was developed, ${ }^{6}$ and refined. ${ }^{7,8}$ In this case, since the projectile velocity is supersonic even for intermediate-energy heavy-ion collisions, a conical shock wave may form, which travels ahead of the projectile through the target nucleus, compressing the target matter. After certain simplifying assumptions, one is able to apply the equations of the oblique-shock-wave problem ${ }^{9-11}$ to fix the flow velocity and the thermodynamical variables immediately behind the conical shock wave.

To determine the full (conical) flow pattern behind the shock, one may argue ${ }^{8}$ that, to some extent, the situation in such an asymmetric collision resembles the case when a bullet (the projectile) moves through a fluid (the target) with supersonic velocity. Thus, the solution of the relativistic Taylor-Maccoll problem ${ }^{12,13}$ may be convenient to describe the flow of nuclear matter behind the conical shock. ${ }^{14}$

In Ref. 7 the oblique-shock-wave problem and in Ref. 8 the relativistic Taylor-Maccoll problem were investigated with the following result: let us assume that, as predicted by QCD lattice simulations, ${ }^{15}$ a first-order phase transition to a deconfined phase, the so-called quark-gluon plasma (QGP), takes place across the shock front. ${ }^{16}$ Then the flow pattern behind the shock is appreciably affected: as measured in the rest frame of the shock, the matter flow is nearly twice as strongly deflected from the original direction ahead of the shock, if a QGP is created as compared to the case where there is ordinary hadronic matter behind the shock wave. It was argued that this might serve as a signal for the deconfinement transition in heavy-ion collisions. The idea is that, if a QGP is created by the conical shock wave above a certain critical bombarding energy, this increase of deflection shows up in the excitation function of the mean deflection angle of matter in asymmetric, central heavy-ion collisions, and thus marks the onset of the deconfinement transition.

The aim of this work is twofold. First, the authors of Refs. 7 and 8 used a particularly simple version of the hadron matter EOS, i.e., an EOS with a constant adiabatic index $\Gamma=(\partial \ln p / \partial \ln n)_{\sigma}(p$ is the pressure, $n$ the net baryon density, and $\sigma$ the specific entropy). This enabled them to obtain most results in analytical terms. In this 
paper we now want to show how more realistic equations of state for hadronic matter [with $\Gamma=\Gamma(n, \sigma)$ ] can be treated in the formalism of the conical-shock-wave problem, i.e., the oblique-shock-wave problem in the vicinity of the shock cone, and the Taylor-Maccoll problem behind the shock. We study their influence on the flow pattern and, in particular, how they affect the importance of the deflection angle as a signature for the phase transition to the QGP. The second aim is the following: since the results are so far obtained in the rest frame of the shock, we transform them to the observer's frame. This is usually the rest frame of the target, i.e., the laboratory. Here one is able to make definite predictions that can be experimentally confirmed. We find that the EOS of the matter under consideration has no influence on the deflection angle of matter in the laboratory frame. Rather, it is the transverse momentum of the matter flow which exhibits the features of the deconfinement transition.

In Sec. II the oblique-shock-wave problem is briefly reviewed and solved for the hadronic equations of state of Refs. 17 and 18. In Sec. III we investigate the TaylorMaccoll problem for the flow of nuclear matter described by the EOS of Ref. 17. The results are compared to the case when a QGP described by the MIT bag EOS is formed across the shock front. ${ }^{7,8}$ In Sec. IV we interpret our results obtained in the rest frame of the shock after transforming them into the laboratory frame and point out some consequences concerning experimental detection. In Sec. V we make some critical remarks concerning the applicability of the discussed picture of a heavyion collision and summarize this work.

\section{THE OBLIQUE-SHOCK-WAVE PROBLEM IN RELATIVISTIC NUCLEAR HYDRODYNAMICS}

Let us assume that the conical shock wave formed in a heavy-ion collision moves with constant speed, given by the bombarding energy, through the target nucleus and that its opening angle $2 \phi$, which is given by the collision geometry, does not change (cf. Fig. 1). ${ }^{14}$ The flow is furthermore assumed to be steady and homogeneous ahead and along both sides of the shock front. Across the shock front it is assumed to be steady. Then, for a given initial state of matter ahead of the shock front and for given $\phi$ and $u_{1}^{\mu}$ (the four-velocity of the target matter relative to the shock front) the equations of ideal relativistic hydrodynamics can be locally (i.e., in the vicinity of the shock front) reduced to the equations of the obliqueshock-wave problem ${ }^{11}$

$$
\begin{aligned}
& {\left[(\epsilon+p) u^{n} u^{0}\right]=0,} \\
& {\left[(\epsilon+p)\left(u^{n}\right)^{2}+p\right]=0,} \\
& {\left[(\epsilon+p) u^{n} u^{t}\right]=0, \quad\left[n u^{n}\right]=0 .}
\end{aligned}
$$

Here $\epsilon, p$, and $n$ are the energy density, the pressure, and the net baryon-number density, respectively. $u^{n}, u^{t}$ are the components of the four-velocity $u^{\mu}=\gamma(1, \boldsymbol{\beta})$ normal and tangential to the shock front ( $\boldsymbol{\beta}$ is the three-velocity) and $u^{0}$ is the time component of $u^{\mu}$. [A] denotes $A_{2}-A_{1}$, where the index 2 refers to the state behind and

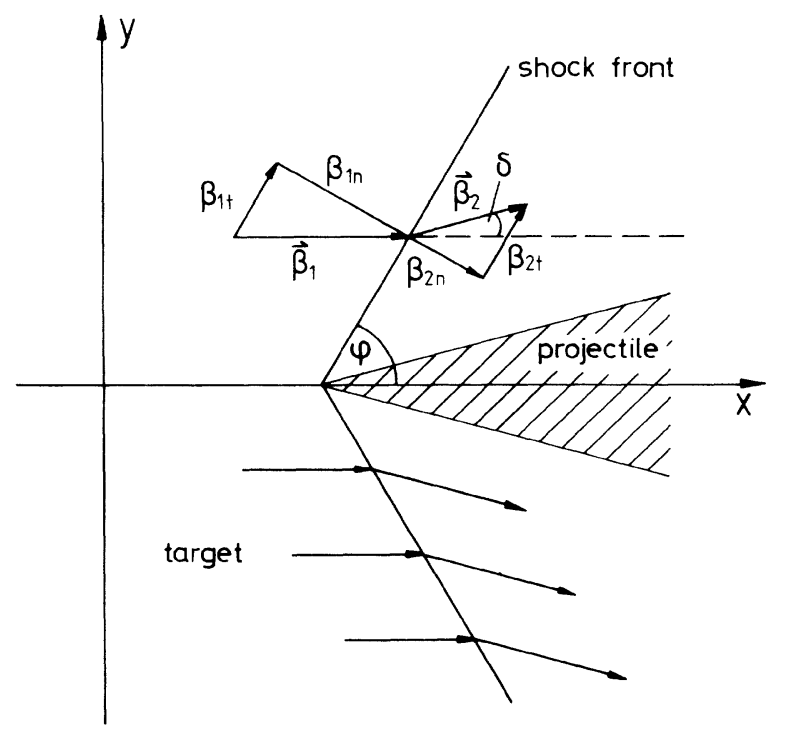

FIG. 1. Schematic picture for the oblique-shock-wave problem (flow along a wedge) to introduce notation used in the text.

1 refers to the state ahead of the shock. Equations (1) relate the initial state of matter and the upstream velocity to the state of matter and the downstream velocity immediately behind the shock front. If we furthermore assume that the flow behind the shock front is steady and homogeneous everywhere [i.e., that matter is characterized everywhere behind the shock front by $\epsilon_{2}, p_{2}, n_{2}, u_{2}^{\mu}$ determined by Eqs. (1) and not only in a small vicinity of the shock], we end up with the hydrodynamical problem of flow along a wedge. ${ }^{9,10}$ We note, however, that for a conical shock geometry one must account for the radial expansion of flow behind the shock front. This is done in Sec. III under the more stringent assumption of conical flow behind the shock. This requires in addition the solution of the relativistic Taylor-Maccoll problem ${ }^{13}$ to determine the state of matter behind the shock front. For the moment, however, let us first discuss the oblique-shockwave problem.

One immediately derives some important consequences from Eqs. (1).

(a) The tangential component of the three-velocity $\beta_{t}$ is continuous across the shock front:

$$
\left[\beta_{t}\right]=0 \text {. }
$$

(b) The Rankine-Hugoniot-Taub-adiabat (RHTA) equation ${ }^{19}$ for plane shocks holds also in this case:

$$
[p]\left(X_{1}+X_{2}\right)=\left[(X n)^{2}\right],
$$

$X=(\epsilon+p) / n^{2}$ is the generalized volume. Therefore, all final states of matter behind the shock front belong to the RHTA of the plane shock problem. The actual final state is, however, not uniquely determined by $u_{1}^{\mu}$ as in the plane case, but depends also on $\phi$.

(c) The ratio of the normal components of the threevelocities $\boldsymbol{\beta}_{1}, \boldsymbol{\beta}_{2}$ is the same as for plane shocks: 


$$
\chi=\frac{\beta_{2, n}}{\beta_{1, n}}=\frac{\epsilon_{1}+p_{2}}{\epsilon_{2}+p_{1}} .
$$

(d) The product of the normal velocities is

$$
\beta_{1, n} \beta_{2, n}=\frac{[p]}{[\epsilon]}\left(1-\beta_{t}^{2}\right) \text {. }
$$

Consequences of this formula for the Mach angle in relativistic flow problems will be discussed below. Note that in the nonrelativistic limit $\beta_{t}<<1$, and we regain the well-known result of Ref. 10 .

(e) Defining

$$
\beta_{0}^{2}=\frac{[p]}{[\epsilon]} \chi^{-1}
$$

$\left(\beta_{0} \equiv \beta_{1} \equiv\left|\beta_{1}\right|\right.$ in the plane shock problem), one is able to express the normal velocities $\beta_{1, n}$ and $\beta_{2, n}$ as functions of $\phi$ and thermodynamical variables ahead of and behind the shock front:

$$
\begin{aligned}
& \beta_{1, n}^{2}=\beta_{0}^{2}\left(1+\beta_{0}^{2} \cot ^{2} \phi\right)^{-1}, \\
& \beta_{2, n}^{2}=\beta_{1, n}^{2} \chi^{2} .
\end{aligned}
$$

Consequences (a)-(e) imply a very simple algorithm to solve the oblique-shock-wave problem for an arbitrary EOS: solving the plane shock problem, i.e., the RHTA equation for a given center $\left(\epsilon_{1}, p_{1}, n_{1}\right)$ one obtains a set of thermodynamic states $\left\{\left(\epsilon_{2}, p_{2}, n_{2}\right)\right\}$. For given $u_{1}^{\mu}$ and $\phi$ one immediately derives $\beta_{t}, \beta_{1, n}$ from geometrical considerations (cf. Fig. 1) and from $\beta_{1}^{2}=u_{1}^{2} /\left(1+u_{1}^{2}\right)$, where $u_{1}^{2}=u_{1, n}^{2}+u_{1, t}^{2}$. According to (7) one consequently knows $\beta_{0}$. One has now only to pick out the state $\left(\epsilon_{2}, p_{2}, n_{2}\right)$ among all solutions of the RHTA equation, which yields, via (6) and (4), a $\beta_{0}$ in agreement with that obtained via (7). Note that different combinations of $u_{1}^{\mu}$ and $\phi$ may yield the same $\beta_{0}$, i.e., the same state $\left(\epsilon_{2}, p_{2}, n_{2}\right)$ behind the shock front. Thus, the physical information contained in the RHTA is not sufficient to uniquely determine the solution of the oblique-shockwave problem.

To this end, it is more convenient to use the so-called "shock-polar" representation. ${ }^{10} \mathrm{We} \mathrm{define}^{7}$

$$
y=\frac{\beta_{2, y}}{\beta_{1}}, \quad x=\frac{\beta_{2, x}}{\beta_{1}} .
$$

From geometrical considerations (cf. Fig. 1) one readily expresses $y$ and $x$ as functions of $\phi$ and $\delta$, the deflection angle of matter behind the shock front:

$$
\begin{aligned}
& y=\frac{\tan \delta}{1+\tan \phi \tan \delta}, \\
& x=\frac{1}{1+\tan \phi \tan \delta} .
\end{aligned}
$$

Note that [from (2)]

$$
\tan \delta=\frac{(1-\chi) \tan \phi}{1+\chi \tan ^{2} \phi}
$$

and thus $\delta$ is completely determined by $\phi$ and $\chi$, which is, in turn, given by the solution of the oblique-shock-wave problem. Hence $y$ and $x$ are uniquely determined by the solution of the oblique-shock-wave problem for given $u_{1}^{\mu}$ and $\phi$. Eliminating $\tan \delta$ from (10) via (11) and $\tan \phi$ between $y$ and $x$ in (10) one obtains

$$
y(x)=\sqrt{(1-\chi)(x-\chi)} \text {. }
$$

Note that $\chi$ is not constant, but depends on the particular solution of the RHTA. Thus, (12) defines in general not a circle but an epicyloid. For given $u_{1}$ the set of points $(y, x)$ is the so-called "shock polar" (cf. Fig. 2). Each point $(y, x)$ on this curve represents a solution of the oblique-shock-wave problem for a different shock angle $\phi$. Another representation of the solution of the obliqueshock-wave problem is to consider $\delta$ as a function of $\phi$ (Ref. 10) (cf. Figs. 3-5). It is completely equivalent to (12), but information about the magnitude of $y$ and $x$ is not directly available [cf. Eq. (10)]. However, an advantage is that the deflection angle of matter can be directly read off. Therefore, we will use this representation in the following.

Let us now present our results for oblique shock waves when the compressed state is hadronic matter. In this case Eq. (3) is the ordinary shock adiabat passing through its center $\left(\epsilon_{1}, p_{1}, n_{1}\right)$, which we take to be the ground state of nuclear matter, $\epsilon_{1}=\epsilon_{0} \simeq 157 \mathrm{MeV} \mathrm{fm}^{-3}, p_{1}=p_{0}=0, n_{1}$ $=n_{0} \simeq 0.17 \mathrm{fm}^{-3}$. To calculate (3), we take the hadronic equations of state of Refs. 17 and 18. The corresponding shock polars in $y-x$ and $\delta-\phi$ representations are shown in Figs. 2-4. Note that the flow velocity behind the shock becomes supersonic if the shock angle is smaller than a certain value $\phi_{\mathrm{cr}}$. Above $\phi_{\mathrm{cr}}$, shocks are called "strong" (solid lines in Figs. 2-4), below they are named "weak" (dotted lines in Figs. 2-4). In the limit $\delta \rightarrow 0, \phi \rightarrow \pi / 2$ (corresponding to $y \rightarrow 0, x<1$ ), we have ordinary strong plane shocks; in the limit $\delta \rightarrow 0, \phi=\phi_{M}<\pi / 2$ (corresponding to $y \rightarrow 0, x \rightarrow 1$ ) the shock becomes merely a sonic disturbance. We note that the velocity of sound for the hadronic equations of state is calculated along the lines given in Ref. 17.

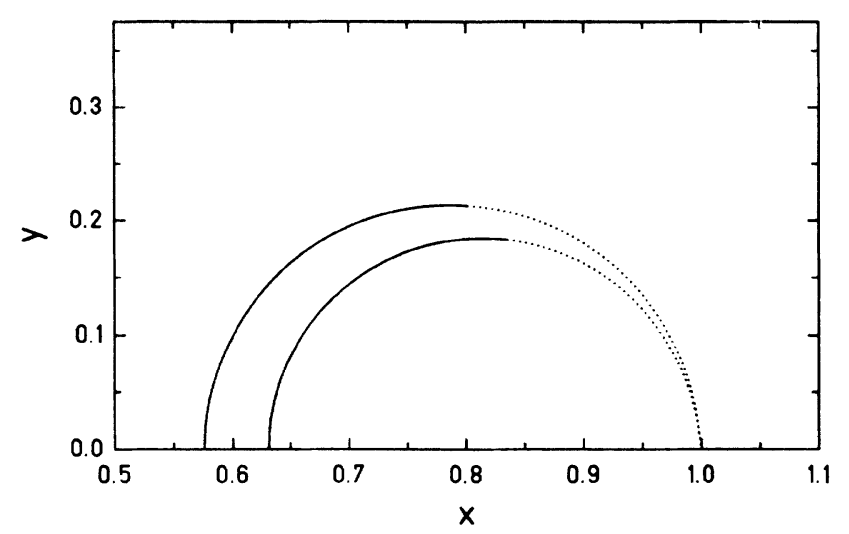

FIG. 2. Shock polars in $y$-x representation for hadronic matter described by the Walecka model $\left(n_{0}=0.15891 \mathrm{fm}^{-3}\right.$, $\mu_{0}=922 \mathrm{MeV}$, lower curve) and the equation of state of Ref. 18 with $K_{0}=300 \mathrm{MeV}$ (upper curve) for $u_{1}^{2}=10$. Dotted lines correspond to supersonic flow behind the shock front, solid lines to subsonic flow. 
For a compression shock adiabat (3), we infer from (5) that, in this case $\left(\beta_{2, n} \rightarrow \beta_{1, n}=\beta_{1} \sin \phi_{M}, \quad[p] /[\epsilon]\right.$ $\rightarrow(d p / d \epsilon)_{0}=\beta_{s, 0}^{2}$ (the velocity of sound in the ground state), $\beta_{t}=\beta_{1} \cos \phi_{M}$ ),

$$
\cos \phi_{M}=\left(1-\frac{\beta_{s, 0}^{2}}{u_{1}^{2}\left(1-\beta_{s, 0}^{2}\right)}\right)^{1 / 2} .
$$

$\phi_{M}$ is the so-called Mach angle. ${ }^{9,10}$ Since

$$
\beta_{s, 0}^{2}=\frac{K_{0}}{9 \mu_{0}} \neq 0
$$

for the equations of state of Refs. 17 and $18\left(K_{0}\right.$ is the ground-state incompressibility and $\mu_{0}$ the chemical potential in the ground state), the curves of Figs. 3 and 4 cannot terminate at the origin (as for the QGP shock polar, see Fig. 5 below), but $\delta$ has to vanish at the finite value $\phi=\phi_{M} \neq 0$. One readily confirms that this value of $\phi_{M}$ in Figs. 3 and 4 is in accord with Eqs. (13) and (14) $\left(K_{0}=300 \mathrm{MeV}, \mu_{0}=922 \mathrm{MeV}\right.$ for the EOS of Ref. 18, $K_{0}=248 \mathrm{MeV}, \mu_{0}=923 \mathrm{MeV}$ for the EOS of Ref. 17).

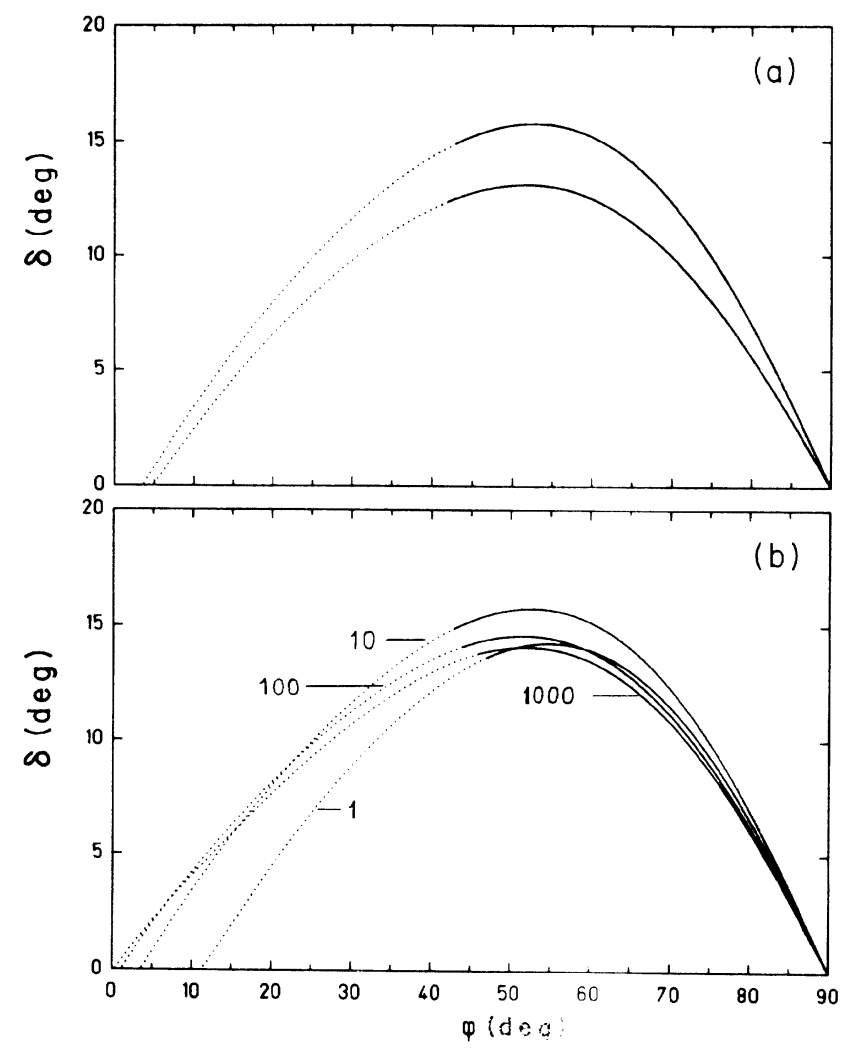

FIG. 3. Shock polars in $\delta-\phi$ representation for hadronic matter described by the Walecka model and the equation of state of Ref. 18 (parameters as in Fig. 2). (a) is the analogue to Fig. 2 (upper curve: EOS of Ref. 18, lower curve: Walecka model); (b) shows the influence of varying $u_{1}^{2}$ for the EOS of Ref. $18\left(u_{1}^{2}=1,10,100,1000\right.$ as indicated in the figure). Dotted lines correspond to supersonic flow behind the shock front, solid lines to subsonic flow.
However, as one reads off Eq. (13), $\phi_{M} \rightarrow 0$ as $u_{1}^{2} \rightarrow \infty$, which is confirmed in Figs. 3 and 4. Let us note that (13) implies

$$
\sin \phi_{M}=\frac{u_{s, 0}}{u_{1}},
$$

with $u_{s, 0}=\beta_{s, 0}\left(1-\beta_{s, 0}^{2}\right)^{-1 / 2}$, and only in the nonrelativistic limit we regain the well-known result $\sin \phi_{M}=\beta_{s, 0} / \beta_{1}$.

In Figs. 3(a) and 4(a) we show the influence of the stiffness of the equations of state under consideration. One observes that for the EOS of Ref. 18 and for the Walecka model the maximum deflection angle is in fair agreement with the results of Ref. 7, obtained with a Bethe-Johnson EOS. However, if the EOS becomes softer (e.g., if we consider the possible excitation of hadronic resonances such as in the EOS of Ref. 17, cf. Fig. 4) the maximum deflection angle increases up to values in the range of that obtained with the MIT bag EOS for the QGP (Fig. 5 and Ref. 7). Since the difference is only a few degrees, the identification of QGP creation in oblique shock waves by means of the deflection angle may cause problems, even in the rest frame of the shock (for the discussion of possible experimental identification, see also Sec. IV).

To conclude this section let us note that the influence

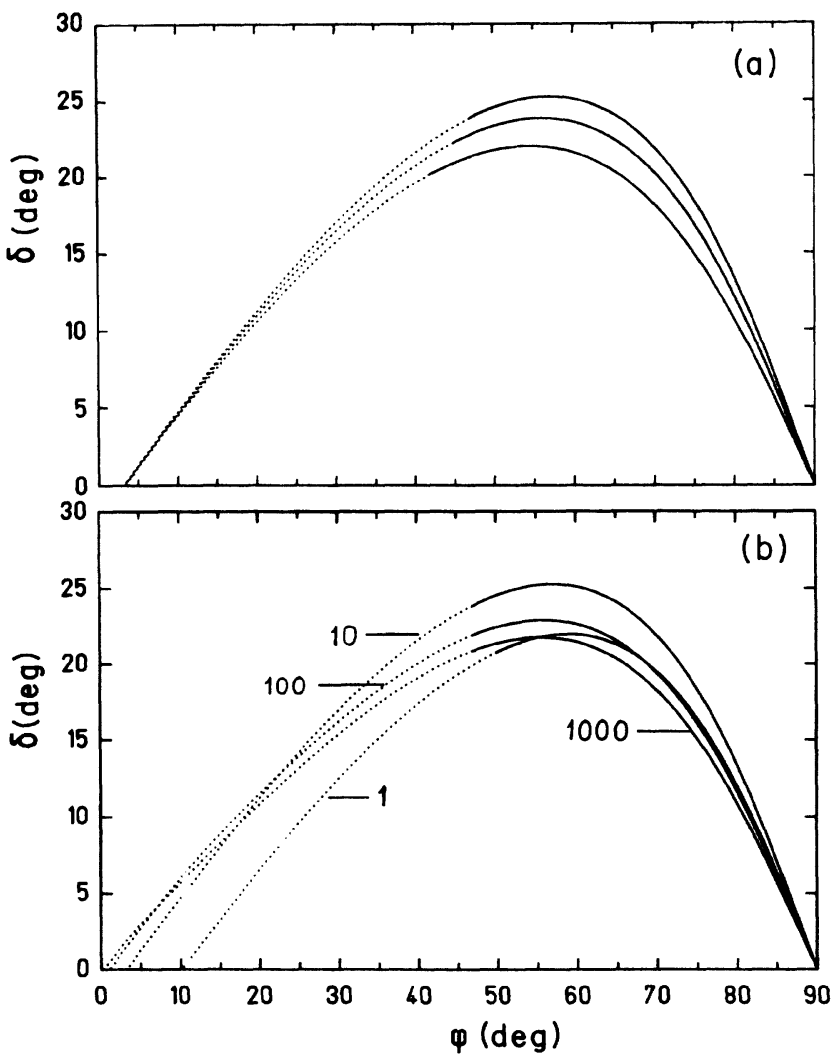

FIG. 4 As in Fig. 3, for the EOS of Ref. 17. (a) shows the influence of the stiffness of the EOS $\left(K_{0}=248,256\right.$, and 266 $\mathrm{MeV}$, from above to below); (b) shows the influence of $u_{1}^{2}$ (for $K_{0}=248 \mathrm{MeV}$ ). 

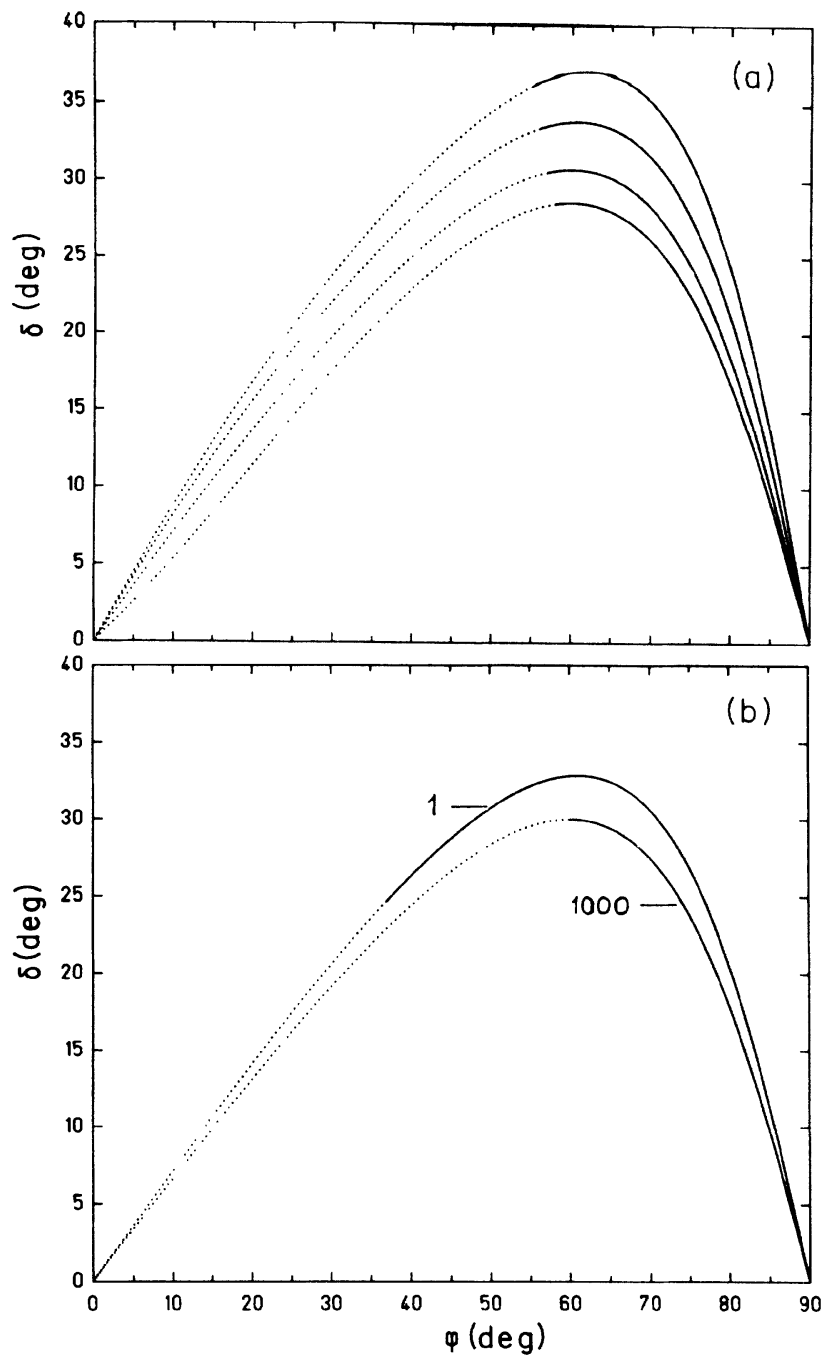

FIG. 5. As in Fig. 3, for the MIT bag EOS. (a) shows the influence of varying the bag constant $B(=400,250,140,80$ $\mathrm{MeV} \mathrm{fm}{ }^{-3}$, from above to below); (b) the influence of $u_{1}^{2}$ (for $B=140 \mathrm{MeV} \mathrm{fm}^{-3}$ ). of the initial upstream velocity $u_{1}$ is rather small as compared to that of the stiffness of the EOS: for the QGP [Fig. 5(b)] we observe that $\delta_{\max }$ decreases monotonously by $\sim 2^{\circ}$ only when $u_{1}$ is increased by an order of magnitude. It changes, however, by $\sim 10^{\circ}$ if we vary the bag constant within the commonly accepted range of values [Fig. 5(a)]. We note that the part of the shock polar corresponding to small $\phi, \delta(x \simeq 1, y<<1)$ is not physical, since it represents unlikely transitions from hadron to quark matter via a small amplitude shock discontinuity. For the hadronic EOS we get similar results: between a very stiff EOS (the Walecka model) and a soft EOS (the EOS of Ref. 17 with $\left.K_{0}=248 \mathrm{MeV}\right) \delta_{\max }$ varies by $\sim 13^{\circ}$ [cf. Figs. 3(a) and 4(a)], while a variation of $u_{1}$ changes $\delta_{\max }$ by only $5^{\circ}$ [cf. Figs. $3(\mathrm{~b})$ and $4(\mathrm{~b})$ ]. Note that $\delta_{\max }$ has a maximum as a function of $u_{1}$ at $u_{1}^{2} \simeq 10$ and then decreases monotonously for all hadronic equations of state under consideration.

\section{THE RELATIVISTIC TAYLOR-MACCOLL PROBLEM}

The Taylor-Maccoll problem is the determination of the hydrodynamical flow pattern of matter moving along an impermeable conical surface. Let us first note that there is, of course, no such object as an impermeable cone in a heavy-ion collision. We rather assume that the flow of nuclear matter in a collision of a small projectile with a large target resembles that of air streaming along a bullet. Whether or not this assumption is viable cannot be proven, but it is very suggestive and leads to an appreciable simplification of the hydrodynamic equations. In Ref. 8 the cone is thought to consist of projectile matter, playing the role of "spectators."

Under certain conditions ${ }^{12,13}$ a conical shock front is attached to the tip of the cone. Thus, for given shock angle $\phi$ one first solves (locally) the oblique-shock-wave problem, as done in Sec. II. Then one accounts for the modification of the flow and the thermodynamical quantities behind the shock front due to the existence of the
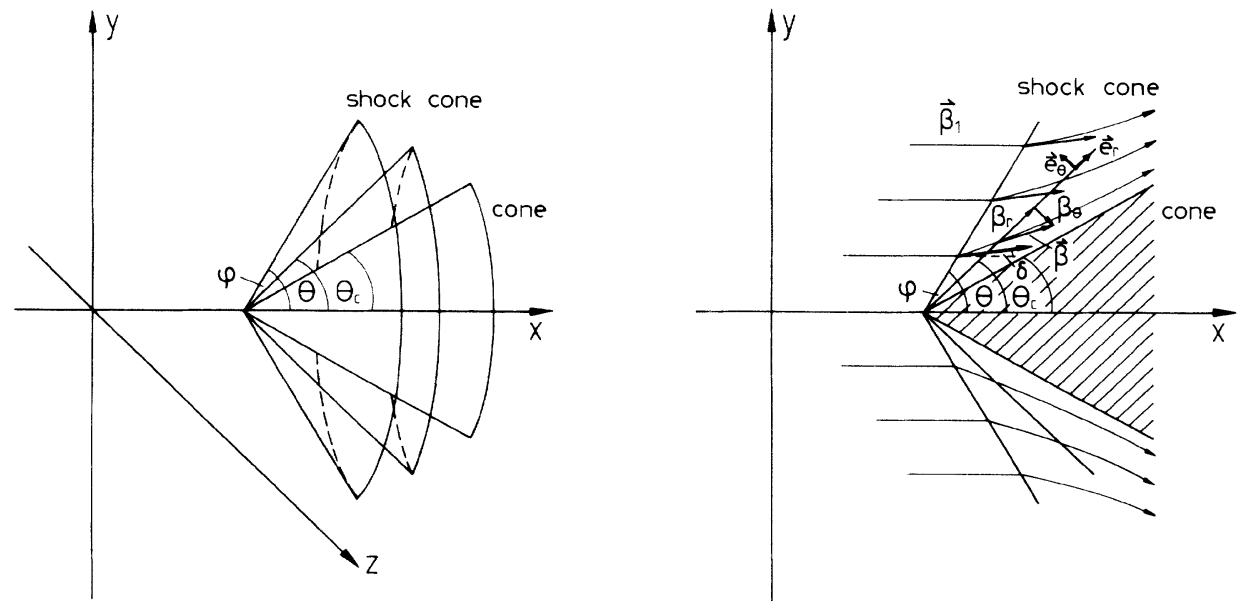

FIG. 6. Schematic picture of the Taylor-Maccoll problem (flow around a cone). 
conical surface. Along this surface the component of the matter velocity normal to the surface vanishes. In physical terms this means that the cone is impermeable.

Matter is still in a steady state behind the shock, but that state is not globally homogeneous. Rather, the flow pattern is azimuthally symmetric and the flow and the properties of the matter are homogeneous ${ }^{12}$ along conical surfaces with opening angles $2 \theta$, with $\theta_{c} \leq \theta \leq \phi$, where $2 \theta_{c}$ is the opening angle of the conical surface (cf. Fig. 6). Thus, in spherical coordinates, all radial derivatives drop out of the hydrodynamical equations. Under the assumption of stationary, isentropic, irrotational flow the hydrodynamic equations reduce to the continuity equation, Bernoulli's equation and the condition for irrotationality:

$$
\begin{aligned}
& \frac{d}{d \theta}\left(n u_{\theta} \sin \theta\right)+2 n u_{\tau} \sin \theta=0, \\
& \frac{\epsilon+p}{n} \gamma=\mathrm{const}, \quad \beta_{\theta}=\frac{d \beta_{r}}{d \theta} .
\end{aligned}
$$

Combining these equations, one derives an ordinary differential equation for the variable $\left(\zeta \equiv \beta_{r}\right.$, the relativistic Taylor-Maccoll equation

$$
\begin{aligned}
& \zeta^{\prime \prime}\left(1+\left(1-\beta_{s}^{-2}\right) \frac{\zeta^{\prime 2}}{1-\zeta^{2}-\zeta^{\prime 2}}\right)+\zeta^{\prime} \cot \theta \\
& +\zeta\left(2+\left(1-\beta_{s}^{-2}\right) \frac{\zeta^{\prime 2}}{1-\zeta^{2}-\zeta^{\prime 2}}\right)=0,
\end{aligned}
$$

where $\beta_{s}$ is the velocity of sound. The result of Ref. 8 is obtained by substituting the value of $\beta_{s}^{2}$ for the BetheJohnson EOS and exploiting Bernoulli's equation. Then a dependence on upstream quantities enters (17). This is, however, not a general feature of the relativistic version of the Taylor-Maccoll equation, as stated in Ref. 8, but is rather due to the special EOS used there.

The problem entering the solution of (17) is that $\beta_{s}$ depends in general on the density $n$. A possible dependence on a second independent thermodynamic variable drops out, since the entropy per baryon $\sigma$ is constant in isentropic flow and thus given by the solution of the obliqueshock-wave problem. The density itself, however, is determined by the continuity equation. Thus, one has to solve simultaneously (17) and the first equation (16). Starting from $\theta=\phi$ one decreases $\theta$ step by step by a small amount until, at a certain value $\theta=\theta_{c}$, the polar coordinate of $\beta$ vanishes, $\beta_{\theta}=\zeta^{\prime}=0$. This is the condition that no matter permeates the conical surface. Thus, the cone angle $\theta_{c}$ is determined. In the original TaylorMaccoll problem ${ }^{12} \theta_{c}$ and $\beta_{r}$ at the cone's surface are given and (17) is integrated from $\theta=\theta_{c}$ until at some value $\theta=\phi$ the state of matter and the velocity coincide with that obtained as solution of the oblique-shock-wave problem. Thus, the shock angle $\phi$ is found.

In Fig. 7(a) we show the solution of the Taylor-Maccoll problem for the hadronic EOS of Ref. 17, i.e., $\beta_{r}$ and $\beta_{\theta}$ behind the shock front as a function of $\theta$ for various upstream velocities $u_{1}$ and a fixed shock angle $\phi=45^{\circ}$. As intuitively expected, $\beta_{\theta}$ increases from its minimum value at the shock front to zero at the cone's surface.
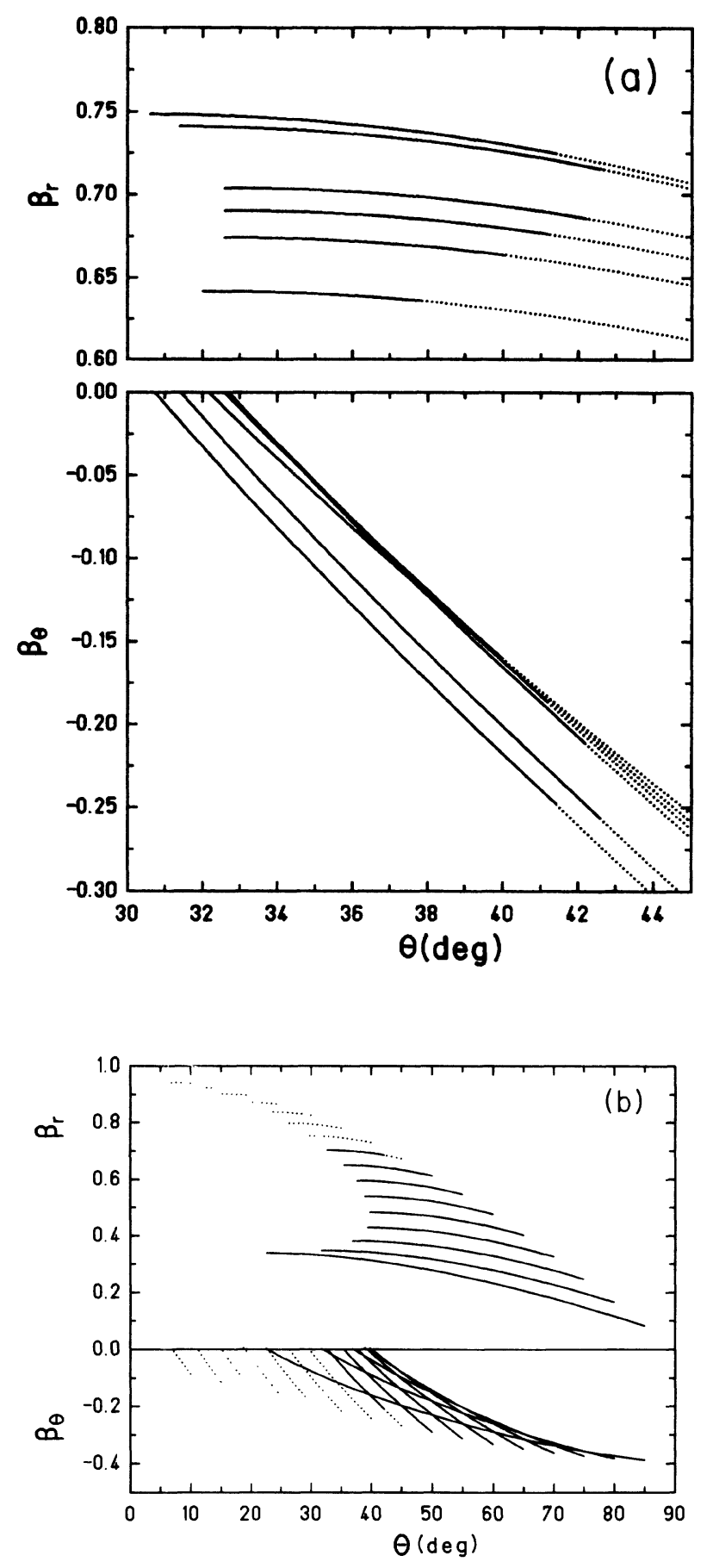

FIG. 7. (a) Radial and tangential velocities as a function of the opening angle $\theta$ (cf. Fig. 6) for the EOS of Ref. 17 with $K_{0}=248 \mathrm{MeV}$. The curves correspond to different values of the upstream velocity $u_{1}$ (in the upper part from below to above: $u_{1}^{2}=3,5,7,10,100,1000$, the corresponding curves in the lower part can be identified by the fact that they terminate at the same $\theta \equiv \theta_{c}$ ) for a shock angle $\phi=45^{\circ}$. Dotted lines: the flow velocity $\beta_{2}=\left(\beta_{r}^{2}+\beta_{\theta}^{2}\right)^{1 / 2}$ is supersonic; solid lines: $\beta_{2}$ is subsonic. The absolute value of the flow velocity decreases when the flow lines approach the cone, due to the pileup of matter along the cone's surface. (b) As in (a), but with fixed $u_{1}^{2}=10$ and for different shock angles (in the upper part from above to below: $\phi=10,15$, $\left.20, \ldots, 80,85^{\circ}\right)$. 
Simultaneously, $\beta_{r}$ increases. However, since the density has to increase towards the cone (there is a pileup of matter along the cone due to the pressure exerted by the cone's surface), the total velocity $\beta_{2}=\left(\beta_{r}^{2}+\beta_{\theta}^{2}\right)^{1 / 2}$ decreases. Hence, the flow may change from supersonic (dotted line) immediately behind the shock front to subsonic (solid line) in the vicinity of the cone.

Observe that there exists a maximum cone angle if $u_{1}^{2} \simeq 10$, which is, of course, related to the fact that for this value the deflection angle $\delta$ immediately behind the shock has a maximum as a function of $u_{1}^{2}$ [cf. Fig. 4(b)].

In Fig. 7(b) we vary the shock angle $\phi$ at fixed $u_{1}$. It is intuitively clear inspecting Fig. 6 that there is a one-toone proportionality between $\theta_{c}$ and $\delta$, a fact that is confirmed in Fig. 7(b). The smaller $\delta$ is, the smaller $\theta_{c}$ should be, although always $\theta_{c}>\delta$. Thus, the statement of Ref. 8 that the cone angle $\theta_{c}$ increases with the shock angle $\phi$ is not correct in general: for large $\phi$ [beyond $\left.\phi\left(\delta_{\max }\right)\right]$, the deflection angle $\delta$, and thus $\theta_{c}$, becomes smaller again (cf. Figs. 3 and 4). We mention that our solution of the Taylor-Maccoll problem for the MIT bag EOS is in agreement with the results of Ref. 8. To summarize this section we note that, provided the flow pattern of matter behind an oblique shock front obeys the Taylor-Maccoll equation, the deflection angle of matter is simply increased as compared to the homogeneous case treated in Sec. III. Thus, if the deflection angle obtained from the solution of the oblique-shock-wave problem for the QGP behind the shock front differs from that obtained with a hadron matter EOS, this difference will be qualitatively preserved in the conical flow.

\section{OBSERVABLE CONSEQUENCES OF QGP FORMATION}

Let us now discuss the results with respect to the experimental identification of the QGP. We first stress that the results of the preceding two sections refer solely to the rest frame of the shock front. Hence, to establish experimentally confirmable predictions we have to transform our results into the laboratory frame. To this end we refer to our picture envisaged in Sec. I that the target is at rest in the laboratory, i.e., that the shock front moves with $u_{1}$ in the $-x$ direction through the target (cf. Fig. 8). ${ }^{14}$ Then, the four-velocity of matter behind the shock front is

$$
\left(u_{2}^{\mu}\right)^{\mathrm{lab}}=\left\{\gamma_{1} \gamma_{2}\left(1-\beta_{1} \beta_{2, x}\right), \gamma_{1} \gamma_{2}\left(\beta_{2, x}-\beta_{1}\right), \gamma_{2} \beta_{2, y}, 0\right\}
$$

and thus

$$
\beta_{2}^{\mathrm{lab}}=\frac{u_{2}^{\mathrm{lab}}}{\gamma_{2}^{\mathrm{lab}}}=\frac{\left\{\beta_{2, x}-\beta_{1}, \beta_{2, y} / \gamma_{1}, 0\right\}}{1-\beta_{1} \beta_{2, x}} .
$$

Hence, the deflection angle $\alpha$ of matter behind an oblique shock front with respect to the beam (i.e., $x$ ) axis is given by

$$
\tan \alpha=\frac{\beta_{2, y}^{\mathrm{lab}}}{\left|\beta_{2, x}^{\mathrm{lab}}\right|}=\gamma_{1}^{-1} \frac{y}{1-x},
$$

with the notations (9). Amusingly enough, because of (10), we obtain

$$
\tan \alpha=\gamma_{1}^{-1} \cot \phi
$$
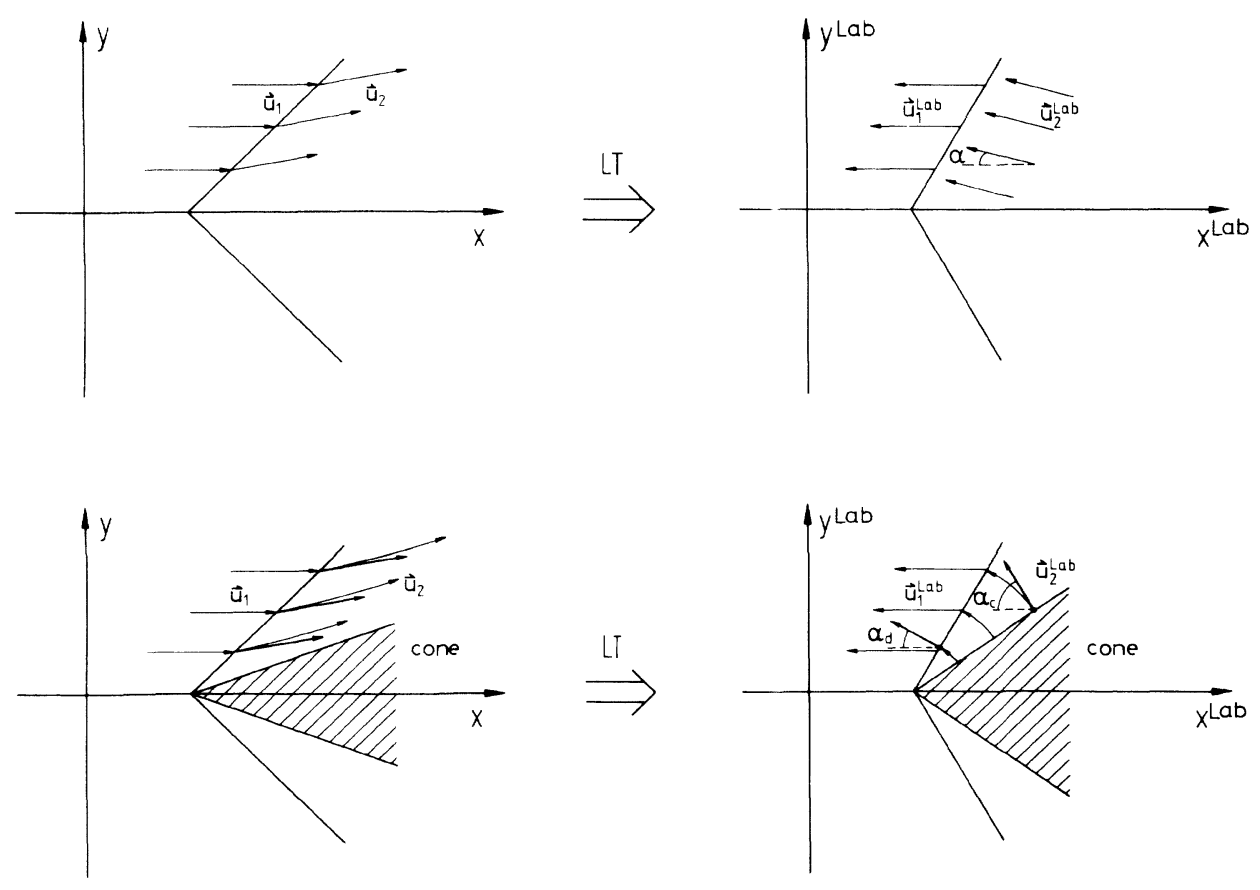

FIG. 8. Schematic picture of the flow in the laboratory system. 
i.e., the deflection of matter in the laboratory frame does not depend on the properties of the matter under consideration, i.e., on the EOS. It solely depends on the shock angle $\phi$ and the velocity of the shock front. Thus, there is no hope to detect the QGP by measuring only the deflection angle behind an oblique shock front for given $u_{1}$ and $\phi$.

This dilemma might be resolved by considering the conical flow behind the shock front. The flow pattern depends on the EOS, entering (17) via $\beta_{s}^{2}$. Thus, the pattern looks different for hadronic matter than for quark matter in the rest frame of the shock. It is very unlikely that these differences also vanish in the laboratory frame. Indeed, Eq. (20) is now replaced by

$$
\tan \alpha=\frac{\beta_{r} \sin \theta-\left|\beta_{\theta}\right| \cos \theta}{\beta_{1}-\beta_{r} \cos \theta-\left|\beta_{\theta}\right| \sin \theta} \gamma_{1}^{-1}
$$

and thus $\tan \alpha$ depends on $\theta$ and also, in contrast with the oblique shock, on the EOS under consideration (via $\beta_{r}$ and $\beta_{\theta}$ ). In Fig. 9 we show for the QGP how the deflection angle of matter immediately behind the shock front $\left(\alpha_{d}\right)$ and in the vicinity of the impermeable cone $\left(\alpha_{c}\right)$ vary as a function of the shock angle $\phi$. One notes that the difference between both, as measured in the laboratory frame, is at most of the order of $2^{\circ}$. This result is fairly independent of the bag constant and the upstream velocity. The same behavior can be found for the hadronic EOS. Thus, there seems little hope to identify the QGP by means of the deflection angle of the matter flow in a conical shock wave in heavy-ion collisions.

However, the deflection angle is not a Lorentzinvariant quantity as, for example, the transverse momentum $p_{\perp}$ of the matter flow. Any difference in $p_{\perp}$, calculated in the rest frame of the shock, would be preserved in the laboratory frame. This is immediately clear noting that $p_{\perp} / M \equiv u_{2, y} \equiv u_{2, y}^{\text {lab }}$ [cf. Eq. (18)]. In general, $M$ denotes the mass of a fluid element and thus an "average" particle mass in the fluid. However, our "fluid" has to

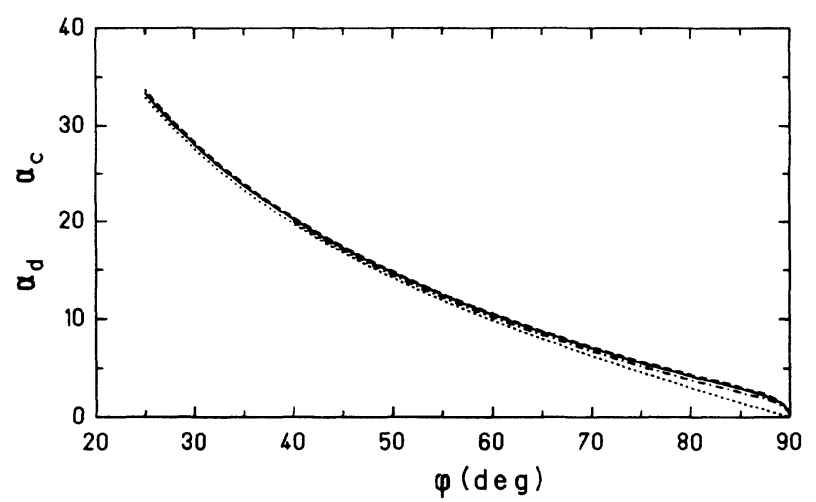

FIG. 9. The deflection angle of quark matter in the laboratory frame for the Taylor-Maccoll problem in the vicinity of the cone $\left(\alpha_{c}\right.$, solid line: $B=140 \mathrm{MeV} \mathrm{fm}^{-3}$, dashed line: $B=80$ $\mathrm{MeV} \mathrm{fm}^{-3}$, dashed-dotted line: $B=400 \mathrm{MeV} \mathrm{fm}^{-3}$ ) and immediately behind the shock front $\left(\alpha_{d}\right.$, dotted line, cf. Fig. 8$)$ as a function of the shock angle $\phi$. "fragment" at freeze-out, before experimental detection is possible. Thus, several particles (mainly pions and nucleons) with different masses and consequently different transverse momenta, but with (nearly ${ }^{20}$ ) the same $p_{\perp} / M$ will enter the detector. It is thus natural to consider the scaled quantity $p_{\perp} / M$ rather than $p_{\perp}$ alone. To get an idea of the order of magnitude of the effects described below one may use $M \sim M_{N} \simeq 1 \mathrm{GeV}$, if the observed particles are nucleons.

In Fig. 10(a) we show $p_{\perp} / M$ versus the shock angle $\phi$ for $u_{1}^{2}=10$ and various equations of state for the oblique-shock-wave problem. One observes that there is a difference in the maximum $p_{\perp}$ of $\sim 100 \mathrm{MeV} / c$ between nucleons emerging from the QGP or from hadronic matter, provided that the hadron matter EOS is not too soft and the bag constant not too small ( $B$ should be in the range of values that produce reasonable deconfinement temperatures $T^{*}$ at vanishing baryon number, ${ }^{15}$ i.e., $T^{*} \simeq m_{\pi} \rightarrow B \simeq 190 \mathrm{MeV} \mathrm{fm}^{-3}$ ).

To make predictions that can be experimentally confirmed, let us express the shock angle $\phi$, which is not an observable quantity, by the kinetic energy of the matter flow in the laboratory frame [Fig. 10(b)]. There is a one-to-one correspondence between $\phi$ and $E_{\text {frag }}^{\text {kin }}$, since $E_{\text {frag }}^{\text {kin }}$ has a maximum for plane shocks $(\phi=\pi / 2)$ and vanishes for $\phi=\phi_{M}$. We first observe that the difference between $p_{\perp} / M$ of quark matter and of hadronic matter is larger for large values of $E_{\mathrm{frag}}^{\mathrm{kin}} / \boldsymbol{M}$. For example, for $E_{\text {frag }}^{\text {kin }} \simeq M$, the transverse momentum of fragments originating from the QGP is even twice as large as that of hadronic matter, which has never undergone a phase transition (if we consider, for instance, $B \simeq 200 \mathrm{MeV} \mathrm{fm}^{-3}$ and the EOS of Ref. 17 with $K_{0}=266 \mathrm{MeV}$ ). We further note that also for given $p_{\perp} / M$ the kinetic energy of nucleons is larger by at least $100 \mathrm{MeV}$, if there is a deconfinement transition across the shock front.

The dependence of the upstream velocity $u_{1}^{2}$ is shown in Fig. 10(c). As is intuitively clear, $p_{\perp} / M$ is larger for larger $u_{1}^{2}$ and assumes its maximum value at larger values of $E_{\text {frag }}^{\text {kin }} / M$.

Considering conical flow behind the shock front does not qualitatively change this behavior. However, the angle of deflection of matter increases in the rest frame of the shock due to the assumed existence of an (impermeable) cone. Hence $u_{2, y} \equiv p_{\perp} / M$ increases in this frame and thus, because of Lorentz invariance, also in the laboratory frame. This increase is of the order of about 0.1 $(\sim 100 \mathrm{MeV} / c$ difference in transverse momentum for nucleons) and fairly independent of the value of the upstream velocity.

From the above it is clear that the creation of a QGP through the conical shock wave exhibits itself by a sudden increase in the excitation function of $p_{\perp} / M$ at fixed $E_{\text {frag }}^{\mathrm{kin}} / M$ (or of $E_{\mathrm{frag}}^{\mathrm{kin}} / M$ at fixed $p_{\perp} / M$ ) at some critical bombarding energy. In this context let us briefly discuss two aspects, which may be of some importance concerning the experimental identification of this effect. Both aspects are related to the following fact: the kinetic quantities $p_{\perp}$ and $E_{\text {frag }}^{\text {kin }}$ are not exactly equal to the mean transverse momentum and the mean kinetic energy of matter 

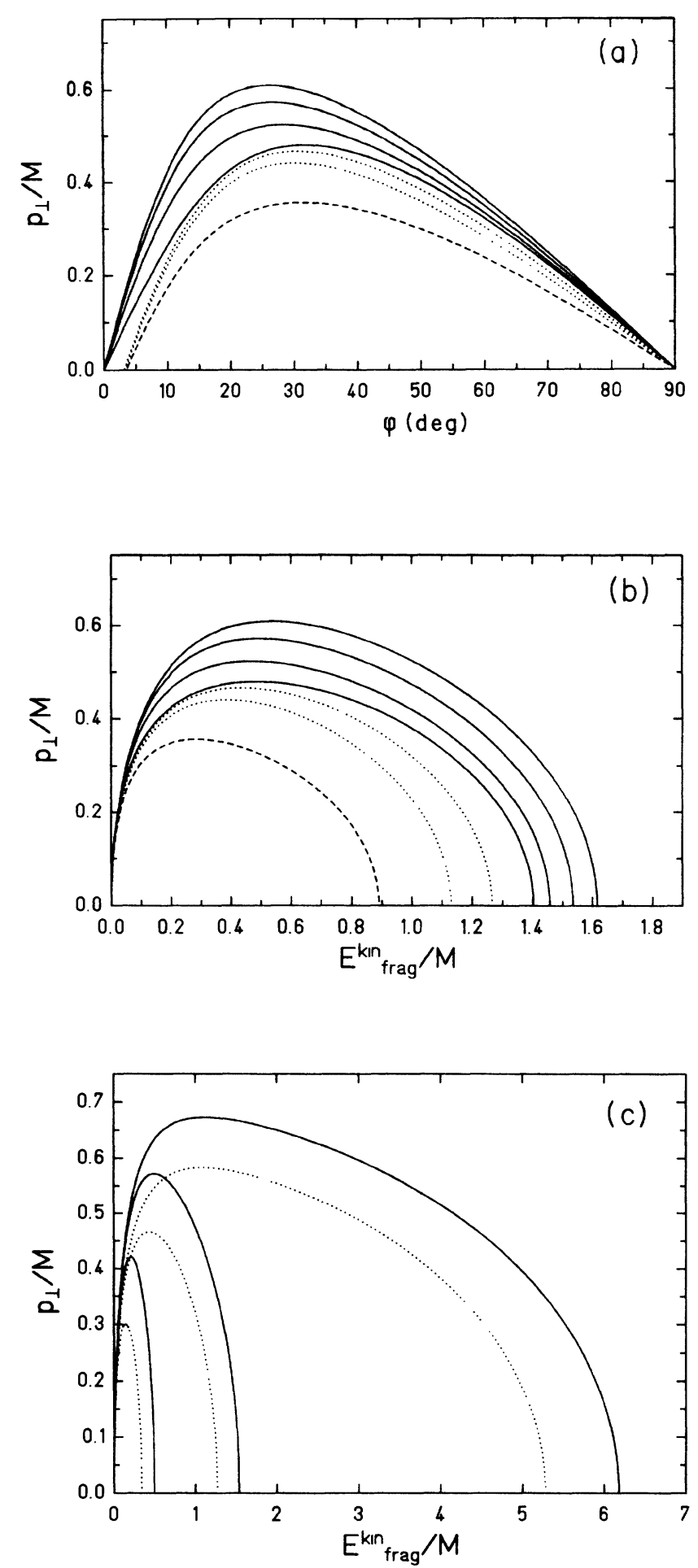

FIG. 10. The transverse momentum of the matter flow behind an oblique shock front as a function of the shock angle $\phi$ (a) and the kinetic energy of fragments in the laboratory frame (b). Solid lines, from above to below: $B=400,250,140,80$ $\mathrm{MeV} \mathrm{fm}^{-3}$. Dotted lines: results for the hadronic EOS of Ref. 17 (upper curve: $K_{0}=248 \mathrm{MeV}$, lower curve: $K_{0}=266 \mathrm{MeV}$ ). Dashed curve: the EOS of Ref. $18\left(K_{0}=300 \mathrm{MeV}\right)$. (c) The dependence of $p_{1} / M$ vs $E_{\mathrm{frag}}^{\mathrm{km}}$ on $u_{1}^{2}$. From below to above: $u_{1}^{2}=2,10,100$, solid line: bag EOS with $B=250 \mathrm{MeV} \mathrm{fm}^{-3}$, dotted line: hadron matter EOS of Ref. $17, K_{0}=248 \mathrm{MeV}$. fragments measured in an experiment. For instance, our quantities neglect the intrinsic thermal motion of the fluid at freeze-out, which essentially broadens the range of possible $p_{1}$ values. However, as a very simple estimate shows, the relative fluctuation of the mean transverse momentum is proportional to $1 / \sqrt{M}$, i.e., for given freeze-out temperature, the relative dispersion of $p_{\perp}$ is half as large for $\alpha$ particles as for nucleons (and only $\sim 1 / 5$ of the dispersion for pions). Thus, the first conclusion is that from the experimental point of view it is advantageous to consider $p_{\perp} / M$ of heavy particles in order to observe an effect of the deconfinement transition.

Second, when estimating the relative fluctuation of $p_{1}$, one also realizes that this quantity is (roughly) inversely proportional to $E_{\text {frag }}^{\text {kin }} / M$. Thus, the relative distortion induced by the temperature is smallest for fragments with large kinetic energy. As we already observed in Fig. 10 (b), the effect of the deconfinement transition is also most dramatic for large $E_{\text {frag }}^{\text {kin }}$. Therefore, the mean transverse momentum of heavy fragments with large kinetic energy is a very promising observable to detect the influence of QGP production on the matter flow.

Of course, more detailed calculations are necessary to account for all effects induced by the freeze-out (cf. also Ref. 21). However, since we do not expect that our simple model is able to make viable quantitative predictions, we are for the moment content to point out qualitative effects of the deconfinement transition on the flow of nuclear matter in heavy-ion collisions.

\section{CONCLUSIONS AND SUMMARY}

In conclusion, let us make some critical remarks concerning the assumptions entering our calculations. In addition to the fact that the viability of the hydrodynamical approach may be limited by principal facts (deviations from local thermodynamical equilibrium may be large), we stress the following points ${ }^{6}$ which are connected with the special picture of a conical shock wave.

(a) If the rate of deceleration of the projectile is of the order of the rate of matter passing through the shock front, the assumption of a uniform shock-front velocity and of a steady flow through this front is violated.

(b) The shock angle may vary in time or space (curved shock fronts), which will in effect introduce some kind of $\phi$ average on the results.

(c) The assumptions entering the shock geometry, e.g., that of a conically shaped shock front, may be too simple. This picture is viable for the flow pattern of a fluid along a "tough" (i.e., "tougher" than the fluid) object, e.g., a bullet in air, as experiments have confirmed. ${ }^{12}$ In our case, however, it would be more reasonable to consider a collision of "drop on drop." Then, however, we are facing the problem that the deceleration of the projectile may be too large, see (a).

(d) Since there is no impermeable object such as a cone in heavy-ion collisions that exerts a force on the fluid, the validity of the Taylor-Maccoll equation is by no means clear. Our assumption that (17) applies relies solely on the very suggestive picture that our asymmetric collision resembles the motion of a bullet in air. 
However, the question whether the conical-shock-wave picture for heavy-ion collisions is too simple and thus inapplicable can only be proved by full $(3+1)$ dimensional calculations. ${ }^{1,2,4,22}$ The intention of this work is simply to confirm that, in a very simple and suggestive picture, ${ }^{7,8}$ there may be principal differences in flow quantities, if a deconfinement transition happens across the shock. These differences, however, cannot be observed measuring the excitation function of the deflection angle, ${ }^{7,8}$ but only via that of the transverse momentum of emitted fragments.

In summary we extended the studies of Refs. 7 and 8 to more realistic nuclear equations of state and investigated the difference between oblique shock waves (and conical flow behind such a wave) in pure hadronic matter and in the case that a QGP is created via such shocks. We found that the softer the hadronic EOS is, the more the flow pattern of such hadronic matter resembles that of quark matter behind the shock front. For given shock velocity and shock angle, there is no effect of the EOS on the deflection angle as measured in the laboratory frame (the rest frame of the matter in front of the shock wave). Assuming conical flow behind the shock wave, one finds that, for a given shock angle $\phi$, the deflection angle of matter immediately behind the shock front and in the vicinity of the cone differs very slightly as measured in the laboratory frame (probably within the experimental accuracy). The effect of the deconfinement transition on the hydrodynamical flow behind a conical shock wave may

${ }^{1}$ H. Stöcker and W. Greiner, Phys. Rep. 137, 277 (1986).

${ }^{2}$ For an overview, see, e.g., R. B. Clare and D. Strottman, Phys. Rep. 141, 177 (1986).

${ }^{3}$ R. Stock, Phys. Rep. 135, 259 (1986).

${ }^{4}$ See, e.g., G. Graebner, Ph.D. thesis, University Frankfurt, 1985; T. Rentzsch, G. Graebner, J. A. Maruhn, H. Stöcker, and W. Greiner, Z. Phys. C 38, 237 (1988); Mod. Phys. Lett. A 2, 193 (1987).

${ }^{5}$ For a general discussion, see, e.g., K. A. Bugaev, M. I. Gorenstein, B. Kämpfer, and V. I. Zhdanov, Phys. Rev. D 40, 2903 (1989).

${ }^{6}$ H. G. Baumgardt, J. U. Schott, Y. Sakamoto, E. Schopper, H. Stöcker, J. Hofmann, W. Scheid, and W. Greiner, Z. Phys. A 273, 359 (1975).

${ }^{7}$ G. F. Chapline and A. Granik, Nucl. Phys. A459, 681 (1986).

${ }^{8}$ G. F. Chapline, A. Granik, Nucl. Phys. A511, 747 (1990).

${ }^{9}$ L. D. Landau and E. M. Lifshitz, Fluid Mechanics (Pergamon, Oxford, England, 1959).

${ }^{10}$ R. Courant and K.O. Friedrichs, Supersonic Flow and Shock Waves (Interscience, New York, 1948).

${ }^{11}$ A. Königl, Phys. Fluids 23, 1083 (1980).

${ }^{12}$ G. I. Taylor and J. W. Maccoll, Proc. R. Soc. A139, 278 (1933); A139, 298 (1933).

${ }^{13}$ A. Granik, Phys. Fluids 29, 1449 (1986). nevertheless be observed, if one considers the excitation function of the transverse momentum of heavy emitted fragments. For instance, $p_{\perp}$ of $\alpha$ particles with a kinetic energy of the order of their mass is larger by $\sim 800$ $\mathrm{MeV} / \mathrm{c}$ if they originate from quark-gluon matter instead of hadronic matter. Thus, in the case of QGP creation, we expect a sharp increase of the excitation function of the mean $p_{\perp}$ at some critical bombarding energy, signaling the onset of the deconfinement transition.

Let us finally mention that another way to identify the creation of a QGP is to perform an event-by-event analysis of heavy-ion collisions. Events with an unusually large $p_{\perp} / M$ in the range of bombarding energies, where the deconfinement transition is expected $(\sim 5-20$ $\mathrm{GeV} /$ nucleon), would also indicate the transient existence of a QGP. We note that in this work a possible coexistence of quark and hadronic matter behind the shock front was not considered. Further investigation along these lines would be interesting, especially with respect to the stability of the shock fronts. ${ }^{5}$ It may clarify the question, why related calculations ${ }^{22}$ show the opposite behavior of the mean transverse momentum in the phase transition region than predicted above.

\section{ACKNOWLEDGMENTS}

The authors gratefully acknowledge helpful discussions with G. F. Chapline, B.L. Friman, M. I. Gorenstein, and A. Granik.
${ }^{14}$ Criticism concerning this assumption is faced in the conclusions.

${ }^{15}$ H. Satz, Annu. Rev. Nucl. Part. Sci. 35, 245 (1985).

${ }^{16}$ U. Heinz, H. Stöcker, and W. Greiner, Proceedings of the Symposium on Relativistic Heavy Ion Research, GSI Darmstadt, 1978 (unpublished); H. Stöcker, G. Graebner, J. A. Maruhn, and W. Greiner, Z. Phys. A 295, 401 (1980); Phys. Lett. 95B, 192 (1980).

${ }^{17}$ D. H. Rischke, B. L. Friman, B. M. Waldhauser, H. Stöcker, and W. Greiner, Phys. Rev. D 41, 111 (1990).

${ }^{18}$ M. I. Gorenstein, D. H. Rischke, K. A. Bugaev, H. Stöcker, and W. Greiner, University of Frankfurt, Institute for Theoretical Phsics Report No. 239/1989 (unpublished).

${ }^{19}$ A. M. Taub, Phys. Rev. 74, 328 (1948).

${ }^{20}$ The freeze-out will affect a thermal and kinematical (Ref. 21) smearing of the transverse momentum, cf. remarks at the end of this section.

${ }^{21}$ M. I. Gorenstein and Yu. M. Sinyukov, Phys. Lett. 142B, 425 (1984).

${ }^{22}$ L. P. Csernai, M. Gong, and D. D. Strottman, Nucl. Phys. A495, 403c (1989); J. A. Zingman, T. L. McAbee, J. R. Wilson, and C. T. Alonso, Phys. Rev. C 38, 760 (1988); T. L. McAbee, J. R. Wilson, J. A. Zingman, and C. T. Alonso, Mod. Phys. Lett. A 4, 983 (1989). 